TAIWANESE JOURNAL OF MATHEMATICS

Vol. 5, No. 2, pp. 279-295, June 2001

Tthis paper is available online at http://www.math.nthu.edu.tw/tjm/

\title{
APPROXIMATION OF DEGENERATE SEMIGROUPS
}

\author{
Wolfgang Arendt
}

\begin{abstract}
By a continuous degenerate semigroup we mean a strongly continuous mapping $T: \mathbb{R}_{+} \rightarrow \mathcal{L}(X)$ having the semigroup property. Thus, $T(0)$ is a projection which may be different from the identity. The main theorem is a Trotter-Kato type approximation result for such degenerate semigroups. It is used to study convergence of heat semigroups with respect to variable domains.
\end{abstract}

\section{INTRODUCTION}

Given an open set $\Omega$ in $\mathbb{R}^{n}$, frequently it is useful to consider the space $L^{2}(\Omega)$ as a subspace of $L^{2}\left(\mathbb{R}^{n}\right)$ extending functions by zero. Given a $C_{0}$-semigroup $T$ on $L^{2}(\Omega)$, the canonical extension of $T$ to $L^{2}\left(\mathbb{R}^{n}\right)$ gives a degenerate continuous semigroup $T_{\Omega}$ on $L^{2}\left(\mathbb{R}^{n}\right)$ such that $T_{\Omega}(0)$ is the orthogonal projection onto $L^{2}(\Omega)$. Given a sequence $\Omega_{k}$ of open sets, we now obtain a sequence of degenerate semigroups on a fixed Hilbert space $L^{2}\left(\mathbb{R}^{n}\right)$ and we may study its convergence. For this, we need an extension of the Trotter-Kato theorem for degenerate semigroups. The Laplace transform of such a semigroup is a pseudoresolvent. Thus, the natural assumption is strong convergence of pseudoresolvents and the aim is to deduce strong convergence of the degenerate semigroups. A most convenient tool is an approximation theorem due to Chernoff [7], which was rediscovered recently by Xiao and Liang [14]. In Section 1, we give a short proof of this result, based merely on the uniqueness theorem for Laplace transforms. Then we use this theorem to study approximation of degenerate semigroups. Under the usual boundedness assumptions one obtains always convergence on the closure of the range space of the limit pseudoresolvent, but not on the kernel as easy examples show. Things are different for uniformly holomorphic sequences, which we consider in Section 5. As application, we study convergence of heat semigroups in Section 6.

Received May 27, 2000; revised September 13, 2000.

Communicated by S.-Y. Shaw.

2000 Mathematics Subject Classification: 47D06, 35K05.

Key words and phrases: Pseudo-resolvent, Trottes-Kato theorem, domain perturbation, convergence of semigroups and resoluents. 


\section{Convergence of Laplace Transforms}

Throughout this article, $X$ denotes a Banach space and $\mathbb{R}_{+}=[0, \infty)$ the closed right half-line. Let $f \in L_{\text {loc }}^{1}\left(\mathbb{R}_{+}, X\right)$ be such that $\left\|\int_{0}^{t} f(s) d s\right\| \leq M e^{\omega t}(t \geq 0)$, where $M, \omega \geq 0$. Then the Laplace transform

$$
\begin{aligned}
\hat{f}(\lambda) & :=\int_{0}^{\infty} e^{-\lambda t} f(t) d t \\
& :=\lim _{\tau \rightarrow \infty} \int_{0}^{\tau} e^{-\lambda t} f(t) d t
\end{aligned}
$$

exists for $\operatorname{Re} \lambda>\omega$ and $\hat{f}:\{\lambda: \operatorname{Re} \lambda>\omega\} \rightarrow X$ is a holomorphic function. The uniqueness theorem asserts the following: If $\hat{f}(\lambda)=0$ for all $\lambda>\lambda_{0}$ and some $\lambda_{0} \geq \omega$, then $f(t)=0$ a.e.

Theorem 1.1 below describes convergence of a sequence of functions in terms of their Laplace transforms. This result was recently proved by Xiao and Liang in [14]. We are grateful to F. Neubrander, who informed us that, in fact, Theorem 1.1 is mentioned by Chernoff [7, p. 106], whose proof of the Proposition on resolvents in [6] carries over to the general situation considered in Theorem 1.1. The special case of Lipschitz continuous functions had been considered by Henning and Neubrander [9].

Here we give a short proof of Theorem 1.1 by applying the uniqueness theorem to the Banach space $\ell^{\infty}(X)$ of all bounded sequences in $X$ with uniform norm.

Theorem 1.1. Let $M, \omega \geq 0$. Let $f_{n}: \mathbb{R}_{+} \rightarrow X$ be continuous and satisfy

$$
\left\|\int_{0}^{t} f_{n}(s) d s\right\| \leq M e^{\omega t} \quad(t \geq 0)
$$

for all $n \in \mathbb{N}$. Assume that

(a) the sequence $\left(f_{n}\right)_{n \in \mathbb{N}}$ is equicontinuous at each $t \geq 0$ and that

(b) $\lim _{n \rightarrow \infty} \hat{f}_{n}(\lambda)$ exists for all $\lambda>\omega$.

Then the sequence $\left(f_{n}\right)_{n \in \mathbb{N}}$ converges uniformly on $[0, \tau]$ for each $\tau>0$.

Proof. Assumption (a) says precisely that $F(t)=\left(f_{n}(t)\right)_{n \in \mathbb{N}}$ defines a continuous mapping $F: \mathbb{R}_{+} \rightarrow \ell^{\infty}(X)$. Since by (1.1)

$$
\left\|\int_{0}^{t} F(s) d s\right\| \leq M e^{\omega t} \quad(t>0)
$$

the function $F$ is Laplace transformable. Denote by $c(X)$ the closed subspace of $\ell^{\infty}(X)$ consisting of all convergent sequences in $X$ and by $q: \ell^{\infty}(X) \rightarrow$ 
$\ell^{\infty}(X) / c(X)$ the quotient map. Then by assumption (b), $\hat{F}(\lambda)=\left(\hat{f}_{n}(\lambda)\right)_{n \in \mathbb{N}} \in$ $c(X)$ for all $\lambda>0$. Now the uniqueness theorem implies that $q \circ F \equiv 0$; i.e., $\left(f_{n}(t)\right)_{n \in \mathbb{N}}$ converges for all $t \in \mathbb{R}_{+}$. Since the sequence is equicontinuous at each $t \in \mathbb{R}_{+}$, simple convergence implies uniform convergence on each compact subset $K$ of $\mathbb{R}_{+}$.

We remark that conditions (a) and (b) are necessary.

If we are merely interested in convergence of $\left(f_{n}(t)\right)_{n \in \mathbb{N}}$ for almost all $t>0$ then the proof of Theorem 1.1 shows that the measurability of $F$ suffices. This is weaker than condition (a), which says precisely that $F$ is continuous. To illuminate this point further, we use the following criterion for measurability, which seems to be new. It is a consequence of Pettis's theorem and the Krein-Smulyan theorem, analogous to Theorem 1.5 below for holomorphic functions.

We use the following terminology. A subset $W$ of the dual space $X^{*}$ of $X$ is called separating if for each $x \in X \backslash\{0\}$ there exists $x^{*} \in W$ such that $\left\langle x^{*}, x\right\rangle \neq 0$.

Theorem 1.2. Let $X$ be a separable Banach space and $f:(0, \infty) \rightarrow X$ a function. Assume that there exists a separating subset $W$ of $X^{*}$ such that $x^{*} \circ f$ is measurable for all $x^{*} \in W$. Then $f$ is measurable.

Proof. Let $Y=\left\{x^{*} \in X^{*}: x^{*} \circ f\right.$ is measurable $\}$. Then $Y$ is a subspace of $X^{*}$ which contains $W$. Thus $Y$ is $\sigma\left(X^{*}, X\right)$-dense in $X^{*}$. Let $Y_{1}=Y \cap B^{*}$, where $B^{*}$ denotes the closed unit ball of $X^{*}$. Since $X$ is separable, the $\sigma\left(X^{*}, X\right)$-topology is metrizable on $B^{*}$. We show that $Y_{1}$ is $\sigma\left(X^{*}, X\right)$-closed. In fact, let $x^{*}$ be an element in the $\sigma\left(X^{*}, X\right)$-closure of $Y_{1}$. Then there exists a sequence $\left(x_{n}^{*}\right)_{n \in \mathbb{N}}$ in $Y_{1}$ converging to $x^{*}$. Hence $\lim _{n \rightarrow \infty}\left\langle x_{n}^{*}, f(t)\right\rangle=\left\langle x^{*}, f(t)\right\rangle$ for all $t>0$. It follows that $x^{*} \circ f$ is measurable; i.e., $x^{*} \in Y_{1}$. Now it follows from the Krein-Smulyan theorem that $Y$ is $\sigma\left(X^{*}, X\right)$-closed in $X^{*}$. Since $Y$ is dense, we have proved that $Y=X^{*}$; i.e., $f$ is weakly measurable. Now Pettis's theorem implies that $f$ is measurable.

With the help of Theorem 1.2 we can easily prove the following:

Corollary 1.3. Let $f_{n}:(0, \infty) \rightarrow X$ be measurable $(n \in \mathbb{N})$ such that

$$
\sup _{\substack{n \in \mathbb{N} \\ t>0}}\left\|f_{n}(t)\right\|<\infty .
$$

Assume that there exists a separable subspace $Y$ of $\ell^{\infty}(X)$ such that $\left(f_{n}(t)\right)_{n \in \mathbb{N}} \in$ $Y$ for almost all $t>0$. If $\left(\hat{f}_{n}(\lambda)\right)_{n \in \mathbb{N}}$ converges for all $\lambda>\omega$ and some $\omega \geq 0$ then $\left(f_{n}(t)\right)_{n \in \mathbb{N}}$ converges for almost all $t>0$. 
Proof. We keep the notations in the proofs of Theorems 1.1 and 1.2. For $x^{*} \in X^{*}$ and $n \in \mathbb{N}$, let $e_{n} \otimes x^{*} \in Y^{*}$ be defined by $\left\langle e_{n} \otimes x^{*}, x\right\rangle=\left\langle x^{*}, x_{n}\right\rangle$, where $x=\left(x_{n}\right)_{n \in \mathbb{N}} \in Y$. Then $W=\left\{e_{n} \otimes x^{*}: n \in \mathbb{N}, x^{*} \in X^{*}\right\} \subset Y^{*}$ is separating and $\left(e_{n} \otimes x^{*}\right) \circ F=x^{*} \circ f_{n}$ is measurable for all $n \in \mathbb{N}, x^{*} \in X^{*}$. It follows from Theorem 1.2 that $F$ is measurable. Now the proof is completed as for Theorem 1.1.

The following example shows that the separability condition cannot be omitted, even in the scalar case.

Example 1.4. Let $X=\mathbb{C}, f_{n}(t)=e^{i n t}$. Then $\hat{f}_{n}(\lambda)=1 /(\lambda-i n) \rightarrow 0$ $(n \rightarrow \infty)$ for all $\lambda>0$; however $\left(f_{n}(t)\right)_{n \in \mathbb{N}}$ does not converge for $t \in \mathbb{R}_{+} \backslash 2 \pi \mathbb{Z}$.

Note that $\left\|\left(f_{n}(s)\right)-\left(f_{n}(t)\right)\right\|_{\ell^{\infty}}=2$ whenever $|s-t| \in \mathbb{R}_{+} \backslash 2 \pi \mathbb{Z}$ so that the separability condition in Corollary 1.3 is not satisfied.

The separability condition in Corollary 1.3 seems difficult to apply in examples. However, a stronger condition, namely holomorphy, is easy to handle. One may use the following criterion [4].

Theorem 1.5. Let $\Omega \subset \mathbb{C}$ be open and $f: \Omega \rightarrow X$ a locally bounded function. Assume that there exists a separating subspace $Y$ of $X^{*}$ such that $y^{*} \circ f$ is holomorphic for all $y^{*} \in Y$. Then $f$ is holomorphic.

Now we can prove the following result.

Theorem 1.6. Let $M, \omega \geq 0$ and let $f_{n}:(0, \infty) \rightarrow X$ be continuous such that $\left\|f_{n}(t)\right\| \leq M e^{\omega t}(t>0)$ for all $n \in \mathbb{N}$. Assume that for each $t>0$ there exists an open disc $B\left(t, \delta_{t}\right)=\left\{z \in \mathbb{C}:|t-z|<\delta_{t}\right\}$, where $\delta_{t}>0$, such that each $f_{n}$ has a holomorphic extension to $B\left(t, \delta_{t}\right)$, still denoted by $f_{n}$, such that

$$
\sup _{n \in \mathbb{N}} \sup _{z \in B\left(t, \delta_{t}\right)}\left\|f_{n}(z)\right\|<\infty
$$

If $\hat{f}_{n}(\lambda)$ converges as $n \rightarrow \infty$ for $\lambda>\omega$, then $f_{n}(t)$ converges as $n \rightarrow \infty$ uniformly on compact intervals of $(0, \infty)$.

Proof. By Theorem 1.5, the function

$$
F(z):=\left(f_{n}(z)\right)_{n \in \mathbb{N}}
$$

is holomorphic on $\Omega:=\bigcup_{t>0} B\left(t, \delta_{t}\right)$ with values in $\ell^{\infty}(X)$. As in the proof of Theorem 1.1, we conclude that $\lim _{n \rightarrow \infty} f_{n}(t)$ exists a.e. It now follows from Vitali's theorem (see [3, Appendix A], [10, pp. 104-105] or [4]) that $\left(f_{n}(z)\right)_{n \in \mathbb{N}}$ converges uniformly on compact subsets of $\Omega$. 


\section{Degenerate Semigroups}

Let $X$ be a Banach space. A degenerate semigroup is a strongly continuous mapping $T:(0, \infty) \rightarrow \mathcal{L}(X)$ satisfying

$$
T(t+s)=T(t) T(s) \quad(s, t>0)
$$

and

$$
\sup _{0<t \leq 1}\|T(t)\|<\infty
$$

If $T(0):=\lim _{t \downarrow 0} T(t)$ exists strongly, we say that $T$ is continuous. Then $T(0)$ is a continuous projection onto $X_{1}=T(0) X$. The restrictions $T(t)_{\left.\right|_{1}}$ define a $C_{0}$-semigroup on $X_{1}$, whereas $T(t)_{\left.\right|_{X_{0}}}=0$ where $X_{0}=(I-T(0)) X$. if

Let $\Omega \subset \mathbb{C}$ be a subset. A function $R: \Omega \rightarrow \mathcal{L}(X)$ is called a pseudoresolvent

$$
\frac{R(\lambda)-R(\mu)}{\mu-\lambda}=R(\lambda) R(\mu) \text { whenever } \mu, \lambda \in \Omega, \mu \neq \lambda .
$$

Then the kernels ker $R(\lambda)$ and the images $R(\lambda) X$ are independent of $\lambda \in \Omega$. If ker $R(\lambda)=\{0\}$, then there exists an operator $A$ on $X$ such that $(\lambda-A)$ is invertible and $R(\lambda)=(\lambda-A)^{-1}$ for all $\lambda \in \Omega$. If $\Omega$ is open, then it follows from (2.3) that $R$ is a holomorphic function.

Conversely, if $\Omega$ is open and connected and $R: \Omega \rightarrow \mathcal{L}(X)$ is holomorphic and if (2.3) is valid for all $\lambda, \mu \in \Omega_{0}, \lambda \neq \mu$, where $\Omega_{0} \subset \Omega$ is a subset of $\Omega$ having a limit point in $\Omega$, then it follows from the uniqueness theorem for holomorphic functions that $R$ is a pseudoresolvent.

Let $T:(0, \infty) \rightarrow \mathcal{L}(X)$ be a degenerate semigroup. Then, as in the case of $C_{0}$-semigroups, there exist $M \geq 0, \omega \in \mathbb{R}$ such that

$$
\|T(t)\| \leq M e^{\omega t} \quad(t>0) .
$$

Thus, we may consider the Laplace transform $\hat{T}(\lambda) \in \mathcal{L}(X)$ of $T$ defined by

$$
\hat{T}(\lambda) x:=\lim _{\tau \rightarrow \infty} \int_{0}^{\tau} e^{-\lambda t} T(t) x d t
$$

for all $x \in X, \lambda \in \mathbb{C}$ such that $\operatorname{Re} \lambda>\omega$. One sees as in [1, Proposition 2.2] or [3, Theorem 3.1.7] that $\hat{T}$ is a pseudoresolvent. Conversely, if $T:(0, \infty) \rightarrow$ 
$\mathcal{L}(X)$ is strongly continuous and satisfies (2.4), and if $\hat{T}:(\omega, \infty) \rightarrow \mathcal{L}(X)$ is a pseudoresolvent, then $T$ is a degenerate semigroup (by the references given above). Similar to the mean ergodic theorem, one has the following decomposition result on reflexive spaces (cf. [13, Corollary VIII.4.1] or [2]). We give a proof for completeness.

Proposition 2.1. Let $R:(\omega, \infty) \rightarrow \mathcal{L}(X)$ be a pseudoresolvent on a reflexive Banach space $X$. Assume that

$$
\limsup _{\lambda \rightarrow \infty}\|\lambda R(\lambda)\|<\infty .
$$

Then $X$ is the direct sum $X=X_{0} \oplus X_{1}$, where $X_{0}=\operatorname{ker} R(\lambda)$ and $X_{1}=\overline{R(\lambda) X}$. Consequently, there exists an operator $A$ on $X_{1}$ such that $(\omega, \infty) \subset \varrho(A)$ and $R(\lambda, A)=R(\lambda)_{\left.\right|_{1}}(\lambda>\omega)$.

\section{Proof.}

(a) Let $\mu>\omega, x=R(\mu) y$. Then $\lambda R(\lambda) R(\mu) y=(\lambda /(\lambda-\mu)(R(\mu) y-R(\lambda) y) \rightarrow$ $R(\mu) y$ as $\lambda \rightarrow \infty$. Hence $\lim _{\lambda \rightarrow \infty} \lambda R(\lambda) x=x$ for all $x \in X_{1}$.

(b) Let $x \in X$ and let $z$ be a $\sigma\left(X, X^{*}\right)$-limit point of $\lambda R(\lambda) x$ as $\lambda \rightarrow \infty$. Then $\left\langle R(\mu) z, x^{*}\right\rangle$ is a limit point of $\left\langle\lambda R(\lambda) x, R(\mu)^{*} x^{*}\right\rangle$ as $\lambda \rightarrow \infty$. Since the last expression converges to $\left\langle R(\mu) x, x^{*}\right\rangle$ as $\lambda \rightarrow \infty$ by a), it follows that $\left\langle R(\mu)(z-x), x^{*}\right\rangle=0$ for all $x^{*} \in X^{*}$. Thus $z-x \in X_{0}$ and $z \in X_{1}$. We have shown that $X \subset X_{0}+X_{1}$.

(c) Since for $x \in X_{1}, \lim _{\lambda \rightarrow \infty} \lambda R(\lambda) x=x$, it follows that $X_{0} \cap X_{1}=\{0\}$.

Corollary 2.2. Each degenerate semigroup on a reflexive space $X$ is continuous.

Proof. Let $T$ be a degenerate semigroup. Then $T$ satisfies (2.4) for some $M \geq 0, \omega \in \mathbb{R}$. Then $\hat{T}:(\omega, \infty) \rightarrow \mathcal{L}(X)$ is a pseudoresolvent. Hence $X=$ $X_{0} \oplus X_{1}$ according to Proposition 2.1. It follows from the uniqueness theorem that $T(t)_{\left.\right|_{0}}=0$. There is an operator $A$ on $X_{1}$ such that $R(\lambda) x=(\lambda-A)^{-1} x$ for $x \in X_{1}, \lambda>\omega$. Thus $D(A)=R(\lambda) X$ is dense in $X_{1}$. It follows from [1, Theorem 2.4] that $A$ satisfies the Hille-Yosida condition. Hence $T(t)_{\left.\right|_{X_{1}}}$ is a $C_{0}$-semigroup.

We mention that on a Banach space $X$ which is not reflexive there may exist a degenerate semigroup which is not continuous even if $X$ has the Radon-Nikodym property (see [1]).

We conclude, showing by an example, that condition (2.5) cannot be omitted in Proposition 2.1. 
Example 2.3. There exists a bounded pseudoresolvent $R:[1, \infty) \rightarrow \mathcal{L}(X)$, where $X$ is a Hilbert space such that $X_{0}+X_{1} \neq X$, where $X_{0}=\operatorname{ker} R(\lambda)$ and $X_{1}=\overline{R(\lambda) X}(\lambda>1)$. In fact, let $\tilde{X}=\left\{x=\left(x_{n}\right)_{n \in \mathbb{N}} \subset \mathbb{C}: \sum_{n=1}^{\infty}\left|x_{n}\right|^{2} /(1+\right.$ $\left.n^{2}\right)<\infty$. Then $\tilde{X}$ is a Hilbert space for the scalar product

$$
(x \mid y)=\sum_{n=1}^{\infty} \frac{x_{n} \cdot \bar{y}_{n}}{1+n^{2}} .
$$

Let $X=\left\{x \in \tilde{X}:\left(x_{2 n}+x_{2 n+1}\right)_{n \in \mathbb{N}} \in \ell^{2}\right\}$, with norm $\|x\|_{X}^{2}=\|x\|_{\tilde{X}}^{2}+\|\left(x_{2 n}+\right.$ $\left.x_{2 n+1}\right)_{n \in \mathbb{N}} \|_{\ell^{2}}^{2}$. Then $X$ is a Hilbert space. Define $R(\lambda) \in \mathcal{L}(X)$ by

$$
(R(\lambda) x)_{n}=\left\{\begin{array}{cl}
\frac{1}{\lambda+n} x_{n} & (n \in 2 \mathbb{N}) \\
0 & n \notin 2 \mathbb{N} .
\end{array}\right.
$$

Then $R:[1, \infty) \rightarrow \mathcal{L}(X)$ is a bounded pseudoresolvent. Consider $u=(1,-1,1,-1$, $\cdots) \in X$. It is obvious that $X_{0}=\left\{x \in X: x_{2 n}=0\right.$ for all $\left.n \in \mathbb{N}\right\}$ and $X_{1} \subset$ $\left\{x \in X: x_{2 n-1}=0\right.$ for all $\left.n \in \mathbb{N}\right\}$. Let $u=(1,-1,1,-1, \cdots) \in X$. Assume that $u=u_{0}+u_{1} \in X_{0}+X_{1}$. Then $u_{0}=(1,0,1,0 \cdots)$ and $u_{1}=(0,-1,0,-1, \cdots)$. But $u_{0} \notin X$ and $u_{1} \notin X_{1}$; a contradiction.

\section{Approximation of Pseudoresolvents}

We start this section by showing that each bounded operator can be embedded into a unique pseudoresolvent. To make this precise, we use the following definition.

Definition 3.1. Let $R: \Omega \rightarrow \mathcal{L}(X)$ be a pseudoresolvent, where $\Omega \subset \mathbb{C}$ is open and connected.

(a) We say that $R$ is maximal if for each pseudoresolvent $R_{1}: \Omega_{1} \rightarrow \mathcal{L}(X)$ with $\Omega_{1}$ an open connected set containing $\Omega$ such that $R_{1}(\lambda)=R(\lambda)$ on $\Omega$, one has $\Omega=\Omega_{1}$.

(b) Let $Q \in \mathcal{L}(X), \lambda_{0} \in \mathbb{C}$. We say that $Q$ is embedded into $R$ at $\lambda_{0}$ if $\lambda_{0} \in \Omega$ and $R\left(\lambda_{0}\right)=Q$.

In the sequel, we will frequently use the following lemma.

Lemma 3.2. Let $R: \Omega \rightarrow \mathcal{L}(X)$ be a pseudoresolvent, where $\Omega \subset \mathbb{C}$ is open.

(a) Let $\lambda \in \Omega$. Then for $\mu \in \Omega$ one has

$$
R(\mu)=(I-(\lambda-\mu) R(\lambda))^{-1} R(\lambda)
$$

whenever $|\lambda-\mu|<\|R(\lambda)\|^{-1}$. 
(b) In particular, $\|R(\mu)\| \leq 2 M$ if $\|R(\lambda)\| \leq M$ and $|\lambda-\mu| \leq(2 M)^{-1}$.

Proof. Since $R(\lambda)-R(\mu)=(\mu-\lambda) R(\lambda) R(\mu)$, one has $R(\lambda)=(I-(\lambda-$ н) $R(\lambda)) R(\mu)$, which implies (3.1). Thus

$$
R(\mu)=R(\lambda) \sum_{n=0}^{\infty}(\lambda-\mu)^{n} R(\lambda)^{n} .
$$

This implies (b).

Next we prove consistency.

Lemma 3.3. Let $\Omega_{1}, \Omega_{2} \subset \mathbb{C}$ be open such that $\Omega_{1} \cap \Omega_{2}$ is connected. Let $\lambda_{0} \in \Omega_{1} \cap \Omega_{2}$. Assume that $R_{j}: \Omega_{j} \rightarrow \mathcal{L}(X)$ are pseudoresolvents $(j=1,2)$ such that $R_{1}\left(\lambda_{0}\right)=R_{2}\left(\lambda_{0}\right)$. Then

(a) $R_{1}(\lambda)=R_{2}(\lambda)$ for all $\lambda \in \Omega_{1} \cap \Omega_{2}$ and

(b)

$$
R(\lambda):= \begin{cases}R_{1}(\lambda) & \left(\lambda \in \Omega_{1}\right) \\ R_{2}(\lambda) & \left(\lambda \in \Omega_{2}\right)\end{cases}
$$

defines a pseudoresolvent on $\Omega_{1} \cup \Omega_{2}$.

Proof. (a) Let $\Omega_{3}=\left\{\lambda \in \Omega_{1} \cap \Omega_{2}: R_{1}(\lambda)=R_{2}(\lambda)\right\}$. Then $\lambda_{0} \in \Omega_{3}$ and $\Omega_{3}$ is relatively closed in $\Omega_{1} \cap \Omega_{2}$ since pseudoresolvents are continuous. Let $\lambda \in \Omega_{3}$. Then $R_{1}(\lambda)=R_{2}(\lambda)$. It follows from Lemma 3.2(a) that $R_{1}(\mu)=R_{2}(\mu)$ if $\mu \in \Omega_{1} \cap \Omega_{2}$ such that $|\mu-\lambda|<\left\|R_{1}(\lambda)\right\|^{-1}$. We have shown that $\Omega_{3}$ is an open, closed and non-empty subset of $\Omega_{1} \cap \Omega_{2}$. Since $\Omega_{1} \cap \Omega_{2}$ is connected, it follows that $\Omega_{3}=\Omega_{1} \cap \Omega_{2}$.

(b) Let $\lambda \in \Omega_{1}$. Set

$$
\Omega_{3}:=\left\{\mu \in \Omega_{2}: \frac{R_{1}(\lambda)-R_{2}(\mu)}{\mu-\lambda}=R_{1}(\lambda) R_{2}(\mu)\right\} .
$$

Then $\Omega_{1} \cap \Omega_{2} \subset \Omega_{3}$ by (a), and hence $\Omega_{3} \neq \emptyset$. We show that $\Omega_{3}$ is open. Let $\mu_{0} \in \Omega_{3}$. Then by Lemma 3.2, for $\left|\mu-\mu_{0}\right|<\left\|R_{2}\left(\mu_{0}\right)\right\|^{-1}$ we have

$$
\begin{aligned}
R_{1}(\lambda)-R_{2}(\mu)= & R_{1}(\lambda)-\left(I-\left(\mu_{0}-\mu\right) R_{2}\left(\mu_{0}\right)\right)^{-1} R_{2}\left(\mu_{0}\right) \\
= & {\left[R_{1}(\lambda)\left(I-\left(\mu_{0}-\mu\right) R_{2}\left(\mu_{0}\right)\right)-R_{2}\left(\mu_{0}\right)\right] } \\
& \left(I-\left(\mu_{0}-\mu\right) R_{2}\left(\mu_{0}\right)\right)^{-1} \\
= & {\left[\left(\mu_{0}-\lambda\right) R_{1}(\lambda) R_{2}\left(\mu_{0}\right)-R_{1}(\lambda)\left(\mu_{0}-\mu\right) R_{2}\left(\mu_{0}\right)\right] } \\
& \left(I-\left(\mu_{0}-\mu\right) R_{2}\left(\mu_{0}\right)\right)^{-1} \\
= & (\mu-\lambda) R_{1}(\lambda) R_{2}\left(\mu_{0}\right)\left(I-\left(\mu_{0}-\mu\right) R_{2}\left(\mu_{0}\right)\right)^{-1} \\
= & (\mu-\lambda) R_{1}(\lambda) R_{2}(\mu) .
\end{aligned}
$$


Hence $\mu \in \Omega_{3}$. Thus $\Omega_{3}$ is open, closed and nonempty. It follows that $\Omega_{3}=\Omega_{2}$. Thus $R$ satisfies the resolvent equation.

Next we embed an operator locally into a pseudoresolvent with the help of (3.1).

Lemma 3.4. Let $Q \in \mathcal{L}(X), M=\|Q\|$, and $\lambda_{0} \in \mathbb{C}$. Define $R(\mu)=$ $\left(I-\left(\lambda_{0}-\mu\right) Q\right)^{-1} Q$ for $\mu \in B\left(\lambda_{0}, 1 / 2 M\right):=\left\{\mu \in \mathbb{C}:\left|\lambda_{0}-\mu\right|<1 / 2 M\right\}$. Then $R$ is a pseudoresolvent.

Proof. Let $\lambda, \mu \in B\left(\lambda_{0}, 1 / 2 M\right)$. Then

$$
\begin{aligned}
R(\lambda)-R(\mu)= & \left(I-\left(\lambda_{0}-\lambda\right) Q\right)^{-1}\left[Q-\left(I-\left(\lambda_{0}-\lambda\right) Q\right) R(\mu)\right] \\
= & \left(I-\left(\lambda_{0}-\lambda\right) Q\right)^{-1}\left[Q\left(I-\left(\lambda_{0}-\mu\right) Q\right)-\left(I-\left(\lambda_{0}-\lambda\right) Q\right) Q\right] \\
& \cdot\left(I-\left(\lambda_{0}-\mu\right) Q\right)^{-1} \\
= & \left(I-\left(\lambda_{0}-\lambda\right) Q\right)^{-1} Q(\mu-\lambda) Q\left(I-\left(\lambda_{0}-\mu\right) Q\right)^{-1} \\
= & (\mu-\lambda) R(\lambda) R(\mu) .
\end{aligned}
$$

Next we characterize maximality.

Proposition 3.5. A pseudoresolvent $R: \Omega \rightarrow \mathcal{L}(X)$ defined on an open connected set $\Omega \subset \mathbb{C}$ is maximal if and only if

$$
\lim _{k \rightarrow \infty}\left\|R\left(\lambda_{k}\right)\right\|=\infty
$$

for each sequence $\left(\lambda_{k}\right)_{k \in \mathbb{N}}$ in $\Omega$ converging to a boundary point $\lambda_{0} \in \partial \Omega$.

Proof. Assume that the condition is not satisfied. Then there exist $\lambda_{0} \in \partial \Omega$ and a sequence $\left(\lambda_{k}\right)_{k \in \mathbb{N}}$ in $\Omega$ converging to $\lambda_{0}$ such that

$$
M:=\sup _{k \in \mathbb{N}}\left\|R\left(\lambda_{k}\right)\right\|<\infty .
$$

Let $k \in \mathbb{N}$ be such that $\left|\lambda_{0}-\lambda_{k}\right|<1 / 2 M$. Then by Lemmas 3.3 and 3.4, $R$ has an extension to $\Omega \cup B\left(\lambda_{k}, 1 / 2 M\right)$ which contains $\lambda_{0}$. Thus $R$ is not maximal. The other implication is obvious since pseudoresolvents are continuous.

Now we can prove the embedding result.

Theorem 3.6. Let $Q \in \mathcal{L}(X)$, and $\lambda_{0} \in \mathbb{C}$. Then there exist an open, connected set $\Omega \subset \mathbb{C}$ and a unique maximal pseudoresolvent $R: \Omega \rightarrow \mathcal{L}(X)$ such that $Q$ is embedded into $R$ at $\lambda_{0}$. 
Proof. The set $I:=\left\{V \subset \mathbb{C}\right.$ open $: \lambda_{0} \in V$, there exists a pseudoresolvent $R_{V}: V \rightarrow \mathcal{L}(X)$ such that $Q$ is embedded into $R_{v}$ at $\left.\lambda_{0}\right\}$ is nonempty by Lemma 3.4. It contains a maximal element by Zorn's lemma. The uniqueness of $R$ follows also from Lemma 3.3.

Using Theorem 3.6, we can prove the following approximation result for pseudoresolvents.

Theorem 3.7. Let $\Omega \subset \mathbb{C}$ be open and connected and let $R_{n}: \Omega \rightarrow \mathcal{L}(X)$ be a pseudoresolvent for each $n \in \mathbb{N}$. Assume that

$$
\sup _{n \in \mathbb{N}}\left\|R_{n}(\lambda)\right\|<\infty
$$

for all $\lambda \in \Omega$. Assume that $\left(R_{n}\left(\lambda_{0}\right)\right)_{n \in \mathbb{N}}$ converges strongly for some $\lambda_{0} \in \Omega$. Then there exists a pseudoresolvent $R: \Omega \rightarrow \mathcal{L}(X)$ such that $R(\lambda) x=\lim _{n \rightarrow \infty} R_{n}(\lambda) x$ uniformly on compact subsets of $\Omega$ for all $x \in X$.

Proof. Let $Q$ be the strong limit of $\left(R\left(\lambda_{0}\right)\right)_{n \in \mathbb{N}}$. Denote by $R: \tilde{\Omega} \rightarrow \mathcal{L}(X)$ the maximal pseudoresolvent such that $Q$ is embedded into $R$ at $\lambda_{0}$ (see Theorem 3.6).

(a) Let $\mu \in \tilde{\Omega} \cap \Omega$. We show that $R(\mu)=\lim _{n \rightarrow \infty} R_{n}(\mu)$ strongly. In fact, let $x \in X$. Let $z=x+\left(\lambda_{0}-\mu\right) R(\mu) x$ and $z_{n}=z+\left(\mu-\lambda_{0}\right) R_{n}\left(\lambda_{0}\right) z$. Then by the resolvent equation, $R(\mu) x=R\left(\lambda_{0}\right) z$ and $R_{n}(\mu) z_{n}=R_{n}\left(\lambda_{0}\right) z$. By hypothesis, $\lim _{n \rightarrow \infty} z_{n}=z+\left(\mu-\lambda_{0}\right) R\left(\lambda_{0}\right) z=z+\left(\mu-\lambda_{0}\right) R(\mu) x=$ $x$. Since $\sup _{n \in \mathbb{N}}\left\|R_{n}(\mu)\right\|<\infty$, it follows that $R(\mu) x=R\left(\lambda_{0}\right) z=$ $\lim _{n \rightarrow \infty} R_{n}\left(\lambda_{0}\right) z=\lim _{n \rightarrow \infty} R_{n}(\mu) z_{n}=\lim _{n \rightarrow \infty}\left[R_{n}(\mu)\left(z_{n}-x\right)+R_{n}(\mu) x\right]$ $=\lim _{n \rightarrow \infty} R_{n}(\mu) x$.

(b) Suppose that $\Omega \not \subset \tilde{\Omega}$. Then there exists $\mu_{0} \in \partial \tilde{\Omega} \cap \Omega$. Let $\mu_{k} \in \tilde{\Omega} \cap \Omega$ be such that $\lim _{k \rightarrow \infty} \mu_{k}=\mu_{0}$. Let $M=\sup _{n \in \mathbb{N}}\left\|R_{n}\left(\mu_{0}\right)\right\|<\infty$. Then, if $\left|\mu_{k}-\mu_{0}\right| \leq 1 / 2 M$, it follows from Lemma 3.2(b) that $\left\|R_{n}\left(\mu_{k}\right)\right\| \leq 2 M$ for all $n \in \mathbb{N}$. Since $\lim _{n \rightarrow \infty} R_{n}\left(\mu_{k}\right)=R\left(\mu_{k}\right)$ strongly, it follows that $\left\|R\left(\mu_{k}\right)\right\| \leq 2 M$. This contradicts the maximality of $R$ by Proposition 3.5. Thus $\Omega \subset \tilde{\Omega}$.

(c) Let $x \in X$. It follows from (a) and (b) that $\lim _{n \rightarrow \infty} R_{n}(\mu) x=R(\mu) x$ for all $\mu \in \Omega$. Lemma 3.2 implies that the sequence $\left(R_{n}\right)_{n \in \mathbb{N}}$ is locally bounded. Thus Vitali's theorem (see [3, Appendix A], [4] or [10, pp. 104-105]) implies that the convergence is uniform on compact subsets of $\Omega$.

Remark 3.8. The proof of (a) shows also the following. Let $\Omega \subset \mathbb{C}$ be an arbitrary set, and $R, R_{n}: \Omega \rightarrow \mathcal{L}(X)$ be pseudoresolvents. Assume that $\sup _{n \in \mathbb{N}}\left\|R_{n}(\lambda)\right\|<\infty$ for all $\lambda \in \Omega$. If there exists some $\lambda_{0} \in \Omega$ such that 
$R\left(\lambda_{0}\right)=\lim _{n \rightarrow \infty} R_{n}\left(\lambda_{0}\right)$ strongly, then $R_{n}(\lambda)$ converges strongly to $R(\lambda)$ for all $\lambda \in \Omega$.

\section{Approximation of Degenerate Semigroups}

In this section, we investigate how strong convergence of degenerate semigroups can be deduced from strong convergence of the associated pseudoresolvents.

We need the following lemma:

Lemma 4.1. Let $T:(0, \infty) \rightarrow \mathcal{L}(X)$ be a degenerate semigroup such that $\|T(t)\| \leq M e^{\omega t}(t>0)$. Let $R(\lambda) x=\int_{0}^{\infty} e^{-\lambda t} T(t) x d t(\lambda>\omega)$. Then

$$
(\lambda R(\lambda)-I) \int_{0}^{t} T(r) d r=(T(t)-I) R(\lambda) \quad(\lambda>\omega, t>0) .
$$

Proof.

$$
\begin{aligned}
(\lambda R(\lambda)-I) \int_{0}^{t} T(r) d r & =\int_{0}^{\infty} \lambda e^{-\lambda s}\left(T(s) \int_{0}^{t} T(r) d r-\int_{0}^{t} T(r) d r\right) d s \\
& =\int_{0}^{\infty} \lambda e^{-\lambda s}\left(\int_{s}^{t+s} T(r) d r-\int_{0}^{t} T(r) d r\right) d s \\
& =\int_{0}^{\infty} \lambda e^{-\lambda s}\left(\int_{t}^{t+s} T(r) d r-\int_{0}^{\infty} T(r) d r\right) d s \\
& =\int_{0}^{\infty} e^{-\lambda s}(T(t+s)-T(s)) d s \\
& =T(t) R(\lambda)-R(\lambda),
\end{aligned}
$$

where integration by parts has been used.

Now we can prove the following approximation result for degenerate semigroups.

Theorem 4.2. Let $T_{n}:(0, \infty) \rightarrow \mathcal{L}(X)$ be a continuous semigroup on a reflexive Banach space $X$ such that

$$
\left\|T_{n}(t)\right\| \leq M e^{\omega t} \quad(t \geq 0)
$$

for all $n \in \mathbb{N}$, where $M, \omega \geq 0$. Denote by $R_{n}(\lambda)=\hat{T}_{n}(\lambda)$ the associated pseudoresolvents $(\lambda>\omega, n \in \mathbb{N})$. Assume that $R_{n}\left(\lambda_{0}\right)$ converges strongly for some $\lambda_{0}>\omega$. Then the following holds.

The Banach space $X$ is the direct sum of subspaces $X_{0}$ and $X_{1}$ such that 
(a) $T(t) x:=\lim _{n \rightarrow \infty} T_{n}(t) x$ converges uniformly on $[0, \tau]$ for all $\tau>0$ and all $x \in X_{1}$, and

(b) $\lim _{n \rightarrow \infty} \int_{0}^{t} T_{n}(s) x d s=0$ uniformly on $[0, \tau]$ for all $\tau>0$ and all $x \in X_{0}$.

Moreover, $T$ is a $C_{0}$-semigroup on $X_{1}$. Let $A_{1}$ be its generator. Then $R\left(\lambda, A_{1}\right) x=$ $\lim _{n \rightarrow \infty} R_{n}(\lambda) x$ for all $x \in X_{1}$ and all $\lambda>\omega$.

Proof. We have the uniform estimate

$$
\left\|R_{n}(\lambda)\right\| \leq \frac{M}{\lambda-\omega} \quad(\lambda>\omega, n \in \mathbb{N}) .
$$

It follows from the hypothesis and Theorem 3.7 that $R(\lambda)=\lim _{n \rightarrow \infty} R_{n}(\lambda)$ exists strongly for all $\lambda>\omega$. Then $(R(\lambda))_{\lambda>\omega}$ is a pseudoresolvent satisfying $\|\lambda R(\lambda)\| \leq$ $M$ for $\lambda \geq \omega+1$. Let $X_{1}=\overline{R(\lambda) X}$ and $X_{0}=$ ker $R(\lambda)$, where $\lambda \geq \omega+1$. Let $y \in X$, and $x=R(\lambda) y$. Let $t \geq 0$. We show that $\left\{T_{n}(\cdot) x\right\}$ is equicontinuous at each $t$. In fact, by Lemma 4.1 one has, for $0<s<t$,

$$
\begin{aligned}
\left\|T_{n}(t) x-T_{n}(s) x\right\|= & \left\|T_{n}(t) R(\lambda) y-T_{n}(s) R(\lambda) y\right\| \\
\leq & \left\|\left(T_{n}(t)-T_{n}(s)\right)\left(R(\lambda) y-R_{n}(\lambda) y\right)\right\|+ \\
& \left\|\left(T_{n}(t)-T_{n}(s)\right) R_{n}(\lambda) y\right\| \\
\leq & \|\left(T_{n}(t)-T_{n}(s)\right)\left(R(\lambda) y-R_{n}(\lambda) y \|+\right. \\
& \left\|\left(T_{n}(t)-I\right) R_{n}(\lambda) y-\left(T_{n}(s)-I\right) R_{n}(\lambda) y\right\| \\
= & \left\|\left(T_{n}(t)-T_{n}(s)\right)\left(R(\lambda) y-R_{n}(\lambda) y\right)\right\| \\
& +\left\|\left(\lambda R_{n}(\lambda)-I\right) \int_{s}^{t} T_{n}(r) y d r\right\| \\
\leq & M\left(e^{\omega t}+e^{\omega s}\right)\left\|R(\lambda) y-R_{n}(\lambda) y\right\| \\
& +(M+1) \int_{s}^{t} M e^{\omega r} d r\|y\| .
\end{aligned}
$$

Given $\varepsilon>0$, we may choose $n_{0} \in \mathbb{N}$ such that the first expression is inferior to $\varepsilon / 2$ for all $n>n_{0}$ and all $0<s \leq t+1$. Now choose $0<\delta<\max \{t, 1\}$ such that the second expression is inferior to $\varepsilon / 2$ whenever $|s-t| \leq \delta$ and also

$$
\left\|T_{n}(t) x-T_{n}(s) x\right\| \leq \varepsilon \text { for } n=1,2, \cdots, n_{0}
$$

if $|s-t| \leq \delta$. Then $\left\|T_{n}(t) x-T_{n}(s) x\right\| \leq \varepsilon$ for all $n \in \mathbb{N}$ whenever $|s-t| \leq \delta$. This shows that the sequence $\left\{T_{n}(\cdot) x: n \in \mathbb{N}\right\}$ is equicontinuous at $t$. Now it follows from Theorem 1.1 that $T_{n}(t) x$ converges uniformly on $[0, \tau]$ as $n \rightarrow \infty$. By density, 
this remains true for all $x \in X_{1}$. Thus (a) is proved. Consequently, $T(t) x:=$ $\lim _{n \rightarrow \infty} T_{n}(t) x\left(x \in X_{1}, t \geq 0\right)$ defines a $C_{0}$-semigroup on $X_{1}$. Moreover, $R\left(\lambda, A_{1}\right) x=\int_{0}^{\infty} e^{-\lambda t} T(t) x d t=\lim _{n \rightarrow \infty} \int_{0}^{\infty} e^{-\lambda t} T_{n}(t) x d t=\lim _{n \rightarrow \infty} R_{n}(\lambda) x$ for all $x \in X_{1}, \lambda>\omega$. In order to show (b), let $S_{n}(t)=\int_{0}^{t} T_{n}(r) d r$. Then $\left\|S_{n}(t)-S_{n}(s)\right\| \leq M \int_{s}^{t} e^{\omega r} d r$ for all $n \in \mathbb{N}$ and $R_{n}(\lambda)=\lambda \int_{0}^{\infty} e^{-\lambda t} S_{n}(t) d t(\lambda>$ $\omega)$. So the set $\left\{S_{n}(\cdot) x: n \in \mathbb{N}\right\}$ is equicontinuous for each $x \in X$. Now it follows from Theorem 1.1 that $\lim _{n \rightarrow \infty} S_{n}(t) x=0$ for all $x \in X_{0}$.

Whereas the integrated semigroups $\int_{0}^{t} T_{n}(r) x d r$ converge as $n \rightarrow \infty$ for each $x \in X$, we have seen in Example 1.4 that it can happen that the semigroups $T_{n}(t) x$ do not converge if $x \in X_{0}$. The situation is better if the semigroups are "uniformly holomorphic" in a sense made precise in the next section.

\section{Holomorphic Degenerate Semigroups}

A bounded holomorphic degenerate semigroup is a degenerate semigroup $T:(0, \infty) \rightarrow X$ which has a bounded holomorphic extension to a sector $\Sigma(\theta):=$ $\left\{r e^{i \alpha}: r>0,|\alpha|<\theta\right\}$ for some $\theta \in(0, \pi / 2]$ with values in $\mathcal{L}(X)$. We also use the letter $T$ for this extension. It follows from the uniqueness of analytic extensions that

$$
T(z) T\left(z^{\prime}\right)=T\left(z+z^{\prime}\right) \text { for all } z, z^{\prime} \in \Sigma(\theta) .
$$

The semigroup is called continuous if $T(t)$ converges strongly as $t \downarrow 0$. By Vitali's theorem, this implies that $T(z)$ converges strongly as $z \rightarrow 0$ for $z \in \Sigma\left(\theta^{\prime}\right)$, for each $\theta^{\prime} \in(0, \theta)$ (cf. [3, Proposition 2.6.3] or [10, Theorem 3.14.3]. By Corollary 2.2, if $X$ is reflexive, then a bounded degenerate holomorphic semigroup is automatically continuous.

Let $T:(0, \infty) \rightarrow \mathcal{L}(X)$ be a bounded holomorphic degenerate semigroup with Laplace transform $\hat{T}(\lambda)(\operatorname{Re} \lambda>0)$. Then one sees as in the $C_{0}$-case that there exists a constant $M \geq 0$ such that

$$
\|\lambda \hat{T}(\lambda)\| \leq M \quad(\operatorname{Re} \lambda>0) .
$$

Conversely, as in the $C_{0}$-case, one proves (e.g., with help of [3, Theorem 2.7.1]) the following converse result. We let $\mathbb{C}_{+}=\{\lambda \in \mathbb{C}: \operatorname{Re} \lambda>0\}$.

Theorem 5.1. Let $M \geq 0$. There exist $\tilde{M} \geq 0, \theta \in(0, \pi / 2]$ such that the following holds. Let $R: \mathbb{C}_{+} \rightarrow \mathcal{L}(X)$ be a pseudoresolvent such that

$$
\|\lambda R(\lambda)\| \leq M \quad(\operatorname{Re} \lambda>0) .
$$


Then there exists a bounded holomorphic degenerate semigroup $T$ such that $\hat{T}(\lambda)=$ $R(\lambda)(\operatorname{Re} \lambda>0)$. Moreover, $T$ has a holomorphic extension to $\Sigma(\theta)$ such that $\|T(z)\| \leq \tilde{M}(z \in \Sigma(\theta))$.

We call $T$ the bounded holomorphic degenerate semigroup associated with $R$.

Now we obtain the following approximation result.

Theorem 5.2. Let $M \geq 0$. For each $m \in \mathbb{N}$, let $R_{m}: \mathbb{C}_{+} \rightarrow \mathcal{L}(X)$ be a pseudoresolvent such that $\left\|\lambda R_{m}(\lambda)\right\| \leq M$ for all $\lambda \in \mathbb{C}_{+}, m \in \mathbb{N}$. Let $T_{m}$ be the bounded holomorphic degenerate semigroup associated with $R_{m}$. If for some $\lambda>$ $0, R_{m}(\lambda)$ converges strongly as $m \rightarrow \infty$, then there exists a bounded holomorphic degenerate semigroup $T$ such that $\lim _{m \rightarrow \infty} T_{m}(t) x=T(t) x$ uniformly on $[0, \tau]$ for all $\tau>0$ and all $x \in X$.

Proof. By Theorem 5.1, there exist $\theta \in(0, \pi / 2], \tilde{M} \geq 0$ such that $T_{m}$ has a holomorphic extension to $\Sigma(\theta)$ with values in $\mathcal{L}(X)$ satisfying $\left\|T_{m}(z)\right\| \leq \tilde{M}$ for all $z \in \Sigma(\theta), m \in \mathbb{N}$. It follows from Theorem 1.6 that $T(z) x:=\lim _{m \rightarrow \infty} T_{m}(z) x$ converges uniformly on compact subsets of $\Sigma(\theta)$ for all $x \in X$. Then $T$ is holomorphic by Vitali's theorem. Moreover, $T\left(z+z^{\prime}\right)=T(z) T\left(z^{\prime}\right)$ for all $z, z^{\prime} \in \Sigma(\theta)$ by the semigroup property of $T_{m}$.

We remark that it is also possible to prove Theorem 5.2 with the help of a contour argument.

\section{Convergence of Heat Semigroups}

Let $\Omega \subset \mathbb{R}^{n}$ be an open set. We identify $L^{2}(\Omega)$ with a closed subspace of $L^{2}\left(\mathbb{R}^{n}\right)$ extending functions by zero. By $\mathcal{D}(\Omega)$ we denote the test functions on $\Omega$ and by $H^{1}\left(\mathbb{R}^{n}\right)$ the first Sobolev space in $L^{2}\left(\mathbb{R}^{n}\right)$. The space $H_{0}^{1}(\Omega)$ is defined as the closure of $\mathcal{D}(\Omega)$ in $H^{1}\left(\mathbb{R}^{n}\right)$. Now we define the Dirichlet Laplacian as the operator $\Delta_{\Omega}$ on $L^{2}(\Omega)$ given by

$$
\begin{aligned}
D\left(\Delta_{\Omega}\right) & =\left\{f \in H_{0}^{1}(\Omega): \Delta f \in L^{2}(\Omega)\right\}, \\
\Delta_{\Omega} f & =\Delta f \text { in } \mathcal{D}(\Omega)^{\prime} .
\end{aligned}
$$

Here we consider $L^{2}(\Omega)$ as a subspace of $\mathcal{D}(\Omega)^{\prime}$ as usual. The operator $\Delta_{\Omega}$ is selfadjoint and form negative, so it generates a holomorphic $C_{0}$-semigroup $\left(e^{t \Delta_{\Omega}}\right)_{t \geq 0}$ on $L^{2}(\Omega)$. We define the degenerate semigroup $T_{\Omega}(t)$ on $L^{2}\left(\mathbb{R}^{n}\right)$ by

$$
\left(T_{\Omega}(t) f\right)(x)=\left\{\begin{array}{lll}
\left(e^{t \Delta_{\Omega}} f_{\left.\right|_{\Omega}}\right)(x) & \text { if } & x \in \Omega \\
0 & \text { if } & x \notin \Omega .
\end{array}\right.
$$


Then $T_{\Omega}$ is continuous and $T_{\Omega}(0)$ is the orthogonal projection onto $L^{2}(\Omega)$ given by $T_{\Omega}(0) f=1_{\Omega} f$.

Definition 6.1 (convergence of open sets). If $\Omega_{m}, \Omega \subset \mathbb{R}^{n}$ are open sets ( $m \in \mathbb{N}$ ), we write $\lim _{m \rightarrow \infty} \Omega_{m}=\Omega$ if the following two conditions are satisfied:

(a) For each compact set $K \subset \Omega$, there exists $m_{0} \in \mathbb{N}$ such that $K \subset \Omega_{m}$ for all $m \geq m_{0}$, and

(b) $\lim _{m \rightarrow \infty}\left|\Omega_{m} \backslash \Omega\right|=0$, where $|F|$ denotes the Lebesgue measure of a measurable subset $F$ of $\mathbb{R}^{n}$.

In the following theorem, we need a mild regularity assumption on the limit set $\Omega$. We let

$$
\tilde{H}_{0}^{1}(\Omega)=\left\{f \in H^{1}\left(\mathbb{R}^{n}\right): f(x)=0 \text { a.e. on } \mathbb{R}^{n} \backslash \Omega\right\} .
$$

Then $\tilde{H}_{0}^{1}(\Omega)$ is a closed subspace of $H^{1}\left(\mathbb{R}^{n}\right)$ containing $H_{0}^{1}(\Omega)$. We will need the assumption that $H_{0}^{1}(\Omega)=\tilde{H}_{0}^{1}(\Omega)$. It is satisfied if the boundary of $\Omega$ is sufficiently regular. For example, if $\Omega$ is bounded and has Lipschitz continuous boundary, then $H_{0}^{1}(\Omega)=\tilde{H}_{0}^{1}(\Omega)$. However, for $\Omega=(0,1) \cup(1,2) \subset \mathbb{R}$ one has $H_{0}^{1}(\Omega) \neq \tilde{H}_{0}^{1}(\Omega)$. We refer to [2] for further details.

Theorem 6.2. Let $\Omega, \Omega_{m} \subset \mathbb{R}^{n}$ be open $(m \in \mathbb{N})$ such that $\lim _{m \rightarrow \infty} \Omega_{m}=\Omega$. Assume that $H_{0}^{1}(\Omega)=\tilde{H}_{0}^{1}(\Omega)$. Then for each $f \in L^{2}\left(\mathbb{R}^{n}\right)$, one has $\lim _{m \rightarrow \infty} T_{\Omega_{m}}(t)$ $f=T_{\Omega}(t) f$ in $L^{2}\left(\mathbb{R}^{n}\right)$ uniformly on $[0, \tau]$ for all $\tau>0$.

Proof. It follows from the spectral theorem that

$$
\left\|\lambda R\left(\lambda, \Delta_{\Omega_{m}}\right)\right\| \leq 1 \quad(\operatorname{Re} \lambda>0)
$$

for all $m \in \mathbb{N}$. Denote by $R_{m}: \mathbb{C}_{+} \rightarrow \mathcal{L}\left(L^{2}\left(\mathbb{R}^{n}\right)\right)$ the pseudoresolvent associated with $T_{\Omega_{m}}$; i.e.,

$$
\left(R_{m}(\lambda) f\right)(x)=\left\{\begin{array}{cc}
\left(R\left(\lambda, \Delta_{\Omega_{m}}\right) f\right)(x) & \left(x \in \Omega_{m}\right), \\
0 & \left(x \notin \Omega_{m}\right),
\end{array}\right.
$$

and let $R$ be the pseudoresolvent associated with $T_{\Omega}$. Let $f \in L^{2}\left(\mathbb{R}^{n}\right), u_{m}=$ $R_{m}(1) f$, and $u=R(1) f$. In order to apply Theorem 5.2 we have to show that $\lim _{m \rightarrow \infty} u_{m}=u$ in $L^{2}\left(\mathbb{R}^{n}\right)$. For this, it suffices to show that each subsequence of $\left(u_{m}\right)_{m \in \mathbb{N}}$ possesses a subsequence which converges to $u$. Note that $u_{m} \in H_{0}^{1}\left(\Omega_{m}\right)$ and $u_{m}-\Delta u_{m}=f$ in $\mathcal{D}\left(\Omega_{m}\right)^{\prime}$; i.e.,

$$
\begin{aligned}
& \int_{\Omega_{m}} u_{m} \varphi+\int_{\Omega_{m}} \nabla u_{m} \nabla \varphi d x= \\
& \int_{\Omega_{m}} u_{m} \varphi-\int_{\Omega_{m}} u_{m} \Delta \varphi d x=\int_{\Omega_{m}} f \varphi d x
\end{aligned}
$$


for all $\varphi \in \mathcal{D}\left(\Omega_{m}\right)$. By density, we deduce that

$$
\int_{\Omega_{m}} u_{m} \varphi+\int_{\Omega_{m}} \nabla u_{m} \nabla \varphi d x=\int_{\Omega_{m}} f \varphi d x
$$

for all $\varphi \in H_{0}^{1}\left(\Omega_{m}\right)$. In particular,

$$
\begin{aligned}
\int_{\Omega_{m}}\left|u_{m}\right|^{2} d x+\int_{\Omega_{m}}\left|\nabla u_{m}\right|^{2} d x & =\int_{\substack{\Omega_{m}\\
}} f u_{m} d x \\
& \leq\|f\|_{L^{2}\left(\mathbb{R}^{n}\right)} \cdot\left\|u_{m}\right\|_{L^{2}\left(\mathbb{R}^{n}\right)} \\
& \leq \frac{1}{2}\left(\|f\|_{L^{2}\left(\mathbb{R}^{n}\right)}^{2}+\left\|u_{m}\right\|_{L^{2}}^{2}\right) .
\end{aligned}
$$

Thus, $\left(u_{m}\right)_{m \in \mathbb{N}}$ is bounded in $H^{1}\left(\mathbb{R}^{n}\right)$. Since $H^{1}\left(\mathbb{R}^{n}\right)$ is reflexive, we may assume that $u_{m}$ converges weakly to a function $v \in H^{1}\left(\mathbb{R}^{n}\right)$ as $m \rightarrow \infty$ (considering a subsequence otherwise). Since for each ball $B$ in $\mathbb{R}^{n}$ the embedding of $H^{1}(B)$ into $L^{2}(B)$ is compact, we can assume that $u_{m}$ converges to $v$ in $L_{\text {loc }}^{2}\left(\mathbb{R}^{n}\right)$ as $m \rightarrow \infty$. So we can also assume that $u_{m}(x)$ converges to $v(x)$ a.e. as $m \rightarrow \infty$ (taking a subsequence again).

Now observe that $\left|u_{m}\right| \leq R(1, \Delta)|f|$ for all $m \in \mathbb{N}$, where $\Delta$ denotes the Laplacian on $L^{2}\left(\mathbb{R}^{n}\right)$, i.e., the generator of the Gaussian semigroup on $L^{2}\left(\mathbb{R}^{n}\right)$ (see [2] for example). Thus it follows from the dominated convergence theorem that $u_{m} \rightarrow v$ in $L^{2}\left(\mathbb{R}^{n}\right)$ as $m \rightarrow \infty$. The assumption b) implies that $1_{\Omega_{m} \backslash \Omega}$ converges to 0 in measure as $m \rightarrow \infty$. As is well-known, this implies that a subsequence converges to 0 a.e. Hence $v(x)=0$ a.e. on $\mathbb{R}^{n} \backslash \Omega$. Thus $v \in \tilde{H}_{0}^{1}(\Omega)=H_{0}^{1}(\Omega)$. Let $\varphi \in \mathcal{D}(\Omega)$. Then there exists $m_{0} \in \mathbb{N}$ such that supp $\varphi \subset \Omega_{m}$ for all $m \geq m_{0}$ (by property a)). Since $u_{m}-\Delta u_{m}=f$ in $\mathcal{D}\left(\Omega_{m}\right)^{\prime}$, it follows that

$$
\int_{\mathbb{R}^{n}} u_{m} \varphi+\int_{\mathbb{R}^{n}} \nabla u_{m} \nabla \varphi=\int_{\mathbb{R}^{n}} f \varphi d x
$$

for all $m \geq m_{0}$. Taking the limit as $m \rightarrow \infty$, we deduce that

$$
\int_{\mathbb{R}^{n}} v \varphi+\int_{\mathbb{R}^{n}} \nabla v \nabla \varphi=\int_{\mathbb{R}^{n}} f \varphi .
$$

We have shown that $v-\Delta v=f$ in $\mathcal{D}(\Omega)^{\prime}$. Thus $v=u$, and the proof is complete.

It is not difficult to generalize Theorem 6.2 to strong convergence in $L^{p}(\Omega)(1 \leq$ $p<\infty)$. In $L^{2}(\Omega)$, one may also use convergence theorems for quadratic forms 
if the sequence of forms is monotonic; see [2], Simon [12] and Reed-Simon [11, Appendix]. Concerning convergence with respect to the domain of the corresponding elliptic problem with different boundary conditions, we refer to Henrot [8] and Bucur [5].

\section{REFERENCES}

1. W. Arendt, Vector-valued Laplace transforms and Cauchy problems. Israel J. Math. 59 (1987), 327-352.

2. W. Arendt and C. Batty, Absorption semigroups and Dirichlet boundary conditions, Math. Ann. 295 (1993), 427-448.

3. W. Arendt, C. Batty, M. Hieber and F. Neubrander, Vector-valued Laplace Transforms and Cauchy Problems, Monographs in Mathematics, Birkhäuser, Basel, to appear.

4. W. Arendt and N. Nikolski, Vector-valued holomorphic functions revisited, Math. Z. 234 (2000), 77-805.

5. D. Bucur, Uniform concentration compactness for Sobolev spaces on variable domains, J. Differential Equations 162 (2000), 427-450.

6. P. R. Chernoff, Note on product formulas for operator semigroups, J. Funct. Anal. 2 (1968), 238-242.

7. P. R. Chernoff, Product Formulas, Nonlinear Semigroups, and Additions of Unbounded Operators, Mem. Amer. Math. Soc. 140 (1974).

8. A. Henrot, Continuity with respect to the domain for the Laplacian: A survey, Control Cybernet. 23 (1994), 427-443.

9. B. Hennig and F. Neubrander, On representations, inversion and approximation of Laplace transforms in Banach spaces, Appl. Anal. 49 (1993), 151-170.

10. E. Hille and R. S. Phillips, Functional Analysis and Semigroups, Amer. Math. Soc. Providence, R.I., 1957.

11. M. Reed and B. Simon, Methods of Modern Mathematical Physics, Vol. 1, Academic Press, New York, 1980.

12. B. Simon, Functional Integration and Quantum Physics, Academic Press, New York, 1979.

13. K. Yosida, Functional Analysis, Springer, Berlin, 1980.

14. Ti-Jun Xiao and Jui Liang, Approximations of Laplace transforms and integrated semigroups, J. Functional Anal. 172 (2000), 221-248.

15. D. Danes, Domain perturbation for linear and nonlinear pasabolic equations. J. Diff. Equ. 129 (1996), 358-402.

Abteilung Angewandte Analysis, Universität Ulm, 89069 Ulm, Germany

E-mail: arendt@mathematik.uni-ulm.de 